\title{
Noise Overexposure Alters Long-Term Somatosensory-Auditory Processing in the Dorsal Cochlear Nucleus-Possible Basis for Tinnitus-Related Hyperactivity?
}

\author{
Susanne Dehmel, Shashwati Pradhan, Seth Koehler, Sanford Bledsoe, and Susan Shore \\ Kresge Hearing Research Institute, University of Michigan, Ann Arbor, Michigan 48109
}

The dorsal cochlear nucleus (DCN) is the first neural site of bimodal auditory-somatosensory integration. Previous studies have shown that stimulation of somatosensory pathways results in immediate suppression or enhancement of subsequent acoustically evoked discharges. In the unimpaired auditory system suppression predominates. However, damage to the auditory input pathway leads to enhancement of excitatory somatosensory inputs to the cochlear nucleus, changing their effects on DCN neurons (Shore et al., 2008; Zeng et al., 2009).

Given the well described connection between the somatosensory system and tinnitus in patients we sought to determine whether plastic changes in long-lasting bimodal somatosensory-auditory processing accompany tinnitus. Here we demonstrate for the first time in vivo long-term effects of somatosensory inputs on acoustically evoked discharges of DCN neurons in guinea pigs. The effects of trigeminal nucleus stimulation are compared between normal-hearing animals and animals overexposed with narrow band noise and behaviorally tested for tinnitus. The noise exposure resulted in a temporary threshold shift in auditory brainstem responses but a persistent increase in spontaneous and sound-evoked DCN unit firing rates and increased steepness of rate-level functions. Rate increases were especially prominent in buildup units. The long-term somatosensory enhancement of sound-evoked responses was strengthened while suppressive effects diminished in noise-exposed animals, especially those that developed tinnitus.

Damage to the auditory nerve is postulated to trigger compensatory long-term synaptic plasticity of somatosensory inputs that might be an important underlying mechanism for tinnitus generation.

\section{Introduction}

In addition to auditory nerve fiber (ANF) innervation, the dorsal cochlear nucleus (DCN) receives somatosensory inputs from head, neck, upper body and limbs via the trigeminal and dorsal column systems, which are transmitted by parallel fibers to synapse on fusiform and cartwheel cells (Itoh et al., 1987; Pfaller and Arvidsson, 1988; Shore et al., 2000; Zhou and Shore, 2004; Haenggeli et al., 2005; Zhan et al., 2006). In normal-hearing animals, paired somatosensory and auditory stimulation leads to suppression or enhancement of acoustically evoked discharges with suppression dominating (Shore, 2005; Shore et al., 2007; Kanold et al., 2011; Koehler et al., 2011). While those studies described the immediate effects of trigeminal and dorsal column stimulation, long-term effects have not been studied in vivo in either normal or noise-damaged systems.

Received Sept. 7, 2011; revised Nov. 23, 2011; accepted Dec. 14, 2011.

Author contributions:S.D. and S.E.S. designed research;S.D., S.P., and S.K. performed research;S.B. contributed unpublished reagents/analytic tools; S.D., S.P., and S.K. analyzed data; S.D. and S.E.S. wrote the paper.

The work was supported by NIH Grants R01DC004825 and P01DC00078, the Tinnitus Research Consortium, and the Tinnitus Research Initiative. We are grateful to Chris Ellinger and Dwayne Vailliencort for technical assistance with the startle and recording setup. We thank Malav Parikh and Kevin Anderson for their invaluable help with data analysis and Ben Yates for excellent graphic assistance. We thank Jeremy Turner for valuable discussions and comments on the manuscript.

Correspondence should be addressed to Susan Shore, Kresge Hearing Research Institute, Departments of Otolaryngology and Molecular and Integrative Physiology, University of Michigan, 1150 W. Medical Center Drive, Ann Arbor, MI 48109. E-mail: sushore@umich.edu.

DOI:10.1523/JNEUROSCI.4608-11.2012

Copyright $\odot 2012$ the authors $\quad 0270-6474 / 12 / 321660-12 \$ 15.00 / 0$
Long-term somatosensory effects accumulating over time could be an important factor in tinnitus development. Auditory nerve damage leads to enhancements of somatosensory inputs and their physiological effects in the DCN (Shore et al., 2008; Zeng et al., 2009). Increased glutamatergic somatosensory innervation and increased sensitivity to those inputs after noise damage might be an underlying mechanism for tinnitus development, with tinnitus being a pathological auditory response to enhanced tonic somatosensory inputs. The connection between tinnitus and the somatosensory system is supported by (1) the observation that tinnitus patients can change the loudness and pitch of their tinnitus by somatosensory manipulations such as contractions or compression of the jaw, head, and neck muscles (Pinchoff et al., 1998; Sanchez et al., 2002; Biesinger et al., 2008), (2) patients can develop tinnitus after somatosensory insults such as tooth abscess, whiplash, and TMJD (Rubinstein et al., 1990; Eggermont, 2005; Levine et al., 2007). Extensive auditory-somatosensory cross-modal interactions are also suggested by the observation that in normal people, auditory sensations can be evoked by contractions of the jaw, head, and neck muscles (Levine et al., 2003).

Here we studied immediate and long-term effects of somatosensory modulation of sound-evoked discharges in DCN neurons in vivo in control and noise-exposed guinea pigs. Results of a behavioral tinnitus test (Turner et al., 2006) were correlated with changes in somatosensory modulation. A comparison between animals with and without tinnitus was made to reveal changes in auditory-somatosensory processing 
as a neuronal correlate and possible underlying cause for tinnitus development.

Noise exposure resulted in a temporary threshold shift (TTS) in auditory brainstem responses (ABRs), but long-term increases in spontaneous and sound-evoked DCN unit firing rates and steeper rate-level functions (RLFs), which were especially prominent in buildup units of tinnitus animals. After paired somatosensoryauditory stimulation, the somatosensory enhancement/suppression of tone-evoked responses is long-term, persisting for up to $135 \mathrm{~min}$ (the longest interval tested). In the noise-exposed animals somatosensory enhancement was strengthened and more units showed enhancement. Chopper as well as buildup units in tinnitus animals showed a strong dominance of long-term enhancement over suppression by somatosensory inputs. This leads to a model of tinnitus development, in which increased excitatory somatosensory influence is triggered by central adjustments to acoustic trauma compensating for the loss of ANF input. The resulting plastic changes persist after hearing threshold recovers. Reversing or counter acting plastic changes in the somatosensory pathways might be an important factor for successful tinnitus therapies.

\section{Materials and Methods}

Animals. Male pigmented guinea pigs from Cady Ridge Farms (300 g at study onset) were used in this study. All procedures were performed in accordance with the National Institutes of Health Guidelines for the Use and Care of Laboratory Animals (NIH publication No. 80-23) and guidelines provided by the University Committee on Use and Care of Animals of the University of Michigan. Of the 20 animals used for DCN recordings, 11 were noise-exposed and 9 non-exposed controls. Eleven animals were tested behaviorally for tinnitus (7 noise-exposed and 4 shamexposed control animals). The numbers of animals contributing to each analysis are indicated in the figure legends.

Gap-detection testing for tinnitus. The gap detection test for tinnitus previously developed for rats (Turner et al., 2006) uses the acoustic startle response elicited by loud pulses and its suppression by preceding substartling prepulses, here gaps in noise bands. It is assumed that ongoing tinnitus masks the gap and results in impaired gap detection (Fig. 1). The gap detection testing was performed using a Kinder Behavioral Testing System. Testing chambers were placed within a single-walled soundproofed booth. Oscillograms of the gap-in-background noise bands and their spectra were examined for reverberations, harmonics and frequency-specificity. The signal spectra outside the gap-carrier/noise pulse bands were between $19 \mathrm{~dB}$ SPL and $24 \mathrm{~dB}$ SPL for each of the frequency bands of the gap-carrier/noise pulses $(4-6 \mathrm{kHz}, 19 \mathrm{~dB}$ SPL; $8-10 \mathrm{kHz}$ and $12-14 \mathrm{kHz}, 23 \mathrm{~dB}$ SPL; $16-18 \mathrm{kHz}, 24 \mathrm{~dB}$ SPL). Gapcarrier/noise pulse bands and startle pulses were calibrated with a microphone (Brüel and Kjaer 1/4 inch 4136), spectrum analyzer (SR760, Stanford Research Systems) and guinea pig cloth model inside the testing cage. A $15 \mathrm{~ms}$ gap (excluding $5 \mathrm{~ms}$ offset/onset ramps) was embedded in four different $2 \mathrm{kHz}$ gap-carrier bands $(4-6,8-10,12-14$, or $16-18 \mathrm{kHz})$ at $70 \mathrm{~dB}$ SPL. The startle pulse ( $115 \mathrm{~dB}$ SPL, BBN, $20 \mathrm{~ms}$ ) followed the onset of the gap by $100 \mathrm{~ms}$. Inter-stimulus trial time (without gap-carrier presentation) was varied pseudo randomly (from 0 to $5 \mathrm{~s}$ ) to prevent anticipation of the startle and interval-based habituation. Detection of the gap results in a reduction in the startle amplitude; development of tinnitus in a specific frequency band impairs gap detection in the corresponding gap-carrier band and thus the startle amplitude reaches amplitudes elicited without the gap (Turner et al., 2006). Animals with normalized startle values (i.e., startle with gap/startle without gap) close to 1 after the noise exposure were grouped together as animals with tinnitus (normalized startles for $8-10 \mathrm{kHz}$ : 0.98 up to 1.46 compared with values between 0.41 and 0.81 for the other animals; see Fig. $4 A$ ). The startles of the noise-exposed group were compared with those of a shamexposed control group. In addition to the gap detection test, animals were also tested with a noise pulse-prepulse inhibition (PPI) test, in which the startle pulse is preceded by a noise pulse at $70 \mathrm{~dB}$ SPL instead of the gap; with the same timing as the gap and the same frequency-

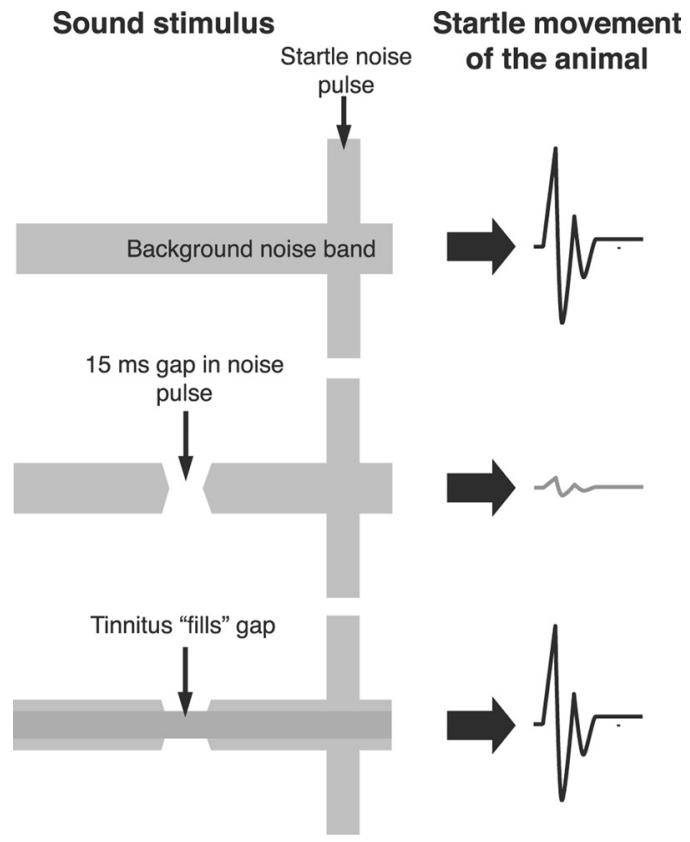

Figure 1. Schematic of the gap detection test. The startle noise pulse elicits a startle movement of the guinea pig (top row) that is reduced when a gap in the background noise band is preceding the startle noise pulse (middle row). Tinnitus that is spectrally similar to the background noise masks the gap and increases the startle movement (bottom row), as the animals cannot detect the gap (Turner et al., 2006).

composition as the gap carrier (see Fig. $4 B$ ). Normal noise pulse detection validates that hearing loss is not the cause for diminished gap-PPI (Yang et al., 2007; Turner and Parrish, 2008). Behavioral testing was done twice a week and continued for the time between the two noise exposures.

Noise exposure. The noise exposure was performed under anesthesia (14 $\mathrm{mg} / \mathrm{kg}$ body weight xylazine, AnaSed Injection, Akorn Inc.; and $110 \mathrm{mg} / \mathrm{kg}$ ketamine, Hospira Inc.; additional dose $4 \mathrm{mg} / \mathrm{kg}$ xylazine and $13 \mathrm{mg} / \mathrm{kg}$ ketamine). Body temperature was kept constant using a temperaturecontrolled heating pad. The left ear was exposed for $2 \mathrm{~h}$ while the right ear was plugged with moldable silicon ear plugs. After the recovery of ABR thresholds, 2 weeks later, a second noise exposure was performed also leading to TTS. The repeated noise exposure was used to increase the incidence of tinnitus. The speaker (Beyer DT 48) was enclosed in a custom-made housing attached to a $3 \mathrm{~cm}$ silicon tube, which ended in a cone-shaped plastic tip that was inserted $2 \mathrm{~mm}$ tightly into the ear canal. The noise was generated with digital signal processing hardware (Tucker-Davis Technologies) and Matlab (MathWorks) and calibrated by inserting the plastic tip into a tube attached to a Brüel and Kjaer $1 / 4$ inch microphone (4136) and spectrum analyzer (SR760, Stanford Research Systems). The noise (see Fig. 3) had a $7 \mathrm{kHz}$ center frequency (CF; maximum set to the geometrical mean) and 1/4 octave bandwidth $(6.4-7.6 \mathrm{kHz})$ with a root mean square level of $97 \mathrm{~dB}$ SPL. Pilot experiments in a separate group of animals showed no influence of the noise on the other ear.

$A B R$ recordings. Before ABRs all animals were routinely injected intramuscularly with an antibacterial solution to prevent infections (enrofloxacin, $10 \mathrm{mg} / \mathrm{kg}$ body weight, Baytril, Bayer). After the ABRs they were injected with $10 \mathrm{ml}$ of saline and treated with antibacterial eardrops (ofloxacin, 0.3\%, Floxin Otic, Daiichi Sankyo Inc.).

ABRs were collected using BioSigRP software and RX5/RA4LI hardware (Tucker-Davis Technologies). The speaker (Beyer DT 48) calibration and acoustic stimulation were performed with SigGenRP software and RX8/PA5 hardware (Tucker-Davis Technologies). The speaker was coupled to the animals' ear canal via a $2 \mathrm{~cm}$ plastic tube inserted into the ear canal. ABRs were recorded immediately before (left ear) and after the noise exposure (left and right ears). ABRs after the exposures were performed weekly on days following the behavioral testing (Tuesdays or Fridays) under anesthesia ( 5 or $10 \mathrm{mg} / \mathrm{kg}$ body weight xylazine and 20 or 

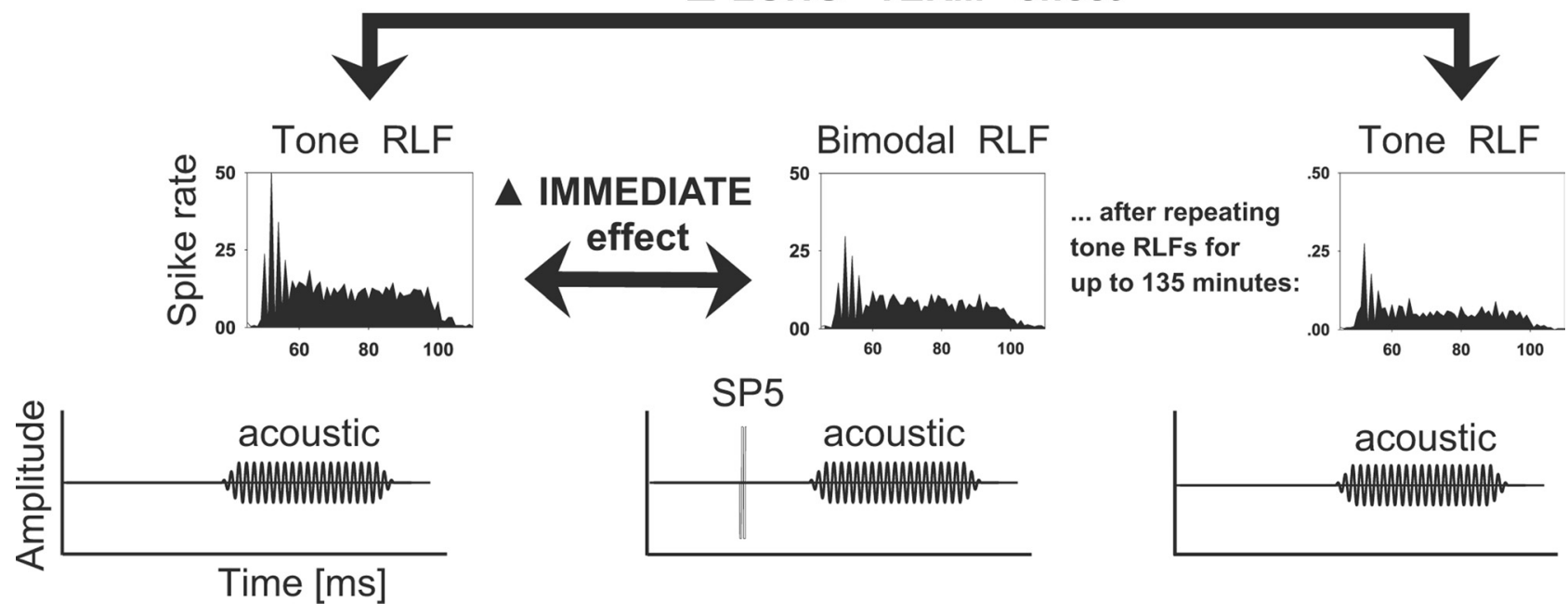

Figure 2. Schematic of the bimodal stimulation paradigm. The immediate effect of bimodal stimulation was derived from a tone RLF and an immediately following bimodal RLF (in which the Sp5 stimulus preceded each tone stimulus). The long-term effect of the bimodal stimulation is derived from the comparison between the first tone RLF and a second tone RLF recorded later in time after the bimodal RLF. For clarity, the stimulus and response are shown only for one tone level of the RLF, which incorporated levels between 0 and 85 in $5 \mathrm{~dB}$ steps with 50 repetitions per level.

$40 \mathrm{mg} / \mathrm{kg}$ body weight ketamine). Body temperature was kept constant with a temperature controlled heating pad. Sanded $0.6 \mathrm{~mm} * 25 \mathrm{~mm}$ injection needles placed subdermally at vertex and on left and right masseter muscles were used for recording, grounding and reference electrodes. ABRs were recorded for $10 \mathrm{~ms}$ tone pips ( $2 \mathrm{~ms}$ ramp, 11 stimuli/s; frequencies $4-16 \mathrm{kHz}$ ) starting at $90 \mathrm{~dB}$ SPL and decremented in $10 \mathrm{~dB}$ steps. Each level was repeated 250 times and the lower levels around threshold were rerun to record a second set of 250 presentations. ABR waveforms were visually inspected across levels; threshold was the lowest level of sound that resulted in one or more of the ABR waves being distinguishable by eye from the background noise. The second set of repetitions for the low levels was checked for consistency of the waveforms.

Extracellular multiunit recordings. DCN recordings were performed $10-21 \mathrm{~d}$ (average $16 \mathrm{~d}$ ) after the second noise exposure. Animals were anesthetized with ketamine $(40 \mathrm{mg} / \mathrm{kg})$ and xylazine $(10 \mathrm{mg} / \mathrm{kg})$ and placed in a stereotaxic frame with hollow ear bars connected to the speakers (Beyer DT770pro). Following bilateral ABR recordings, a craniotomy and partial aspiration of the cerebellum overlying the DCN was performed to allow visual placement of the probes (4 shank/16 channel silicon, $177 \mu \mathrm{m}^{2}$ sites, pitch $100 \mu \mathrm{m}$, NeuroNexus) between 300 and 500 $\mu \mathrm{m}$ below the DCN surface. Stimulation and multiunit recordings were achieved using OpenEx software (Tucker-Davis Technologies), which controlled RX8 DSP and RX5 respectively. Thresholds and center frequencies (CFs) were determined online (OpenExplorer, Tucker-Davis Technologies) from a receptive field generated from 10 repetitions of a 50 $\mathrm{ms}$ duration tone (200 ms stimulation period) at each frequency-level condition ( 0.1 octave steps between $100 \mathrm{~Hz}$ and $36 \mathrm{kHz}, 5 \mathrm{~dB}$ steps $0-85$ $\mathrm{dB}$ SPL). RLFs were generated for 50 repetitions of each $5 \mathrm{~dB}$ step. To assess immediate effects of bimodal stimulation, a tone RLF (a series of tone burst stimuli between 0 and $85 \mathrm{~dB}$ ) was immediately followed by a bimodal RLF (electric Sp5 stimulation preceding each tone stimulus by $20 \mathrm{~ms}$ ). To assess long-term effects, a tone RLF was followed by a bimodal RLF and then tone RLFs were subsequently repeated for up to $135 \mathrm{~min}$ (the longest time interval tested, minimum time interval 15 min, mean 35 min; Fig. 2). In some animals, RLFs were recorded before and after Sp5 stimulation alone to obtain control data (data not shown) that indicated that the long-term effect was due to paired stimulation and not Sp5 stimulation alone. Poststimulus time histogram type was determined from the tone RLFs $20-40 \mathrm{~dB}$ above threshold and then averaged across units of all animals and within the groups of control, tinnitus and notinnitus animals.

The caudal and intermediate regions of Sp5 were electrically stimulated with a custom-made isolated high-voltage current source via concentric bipolar electrodes (FHC) placed stereotaxically (Koehler et al.,

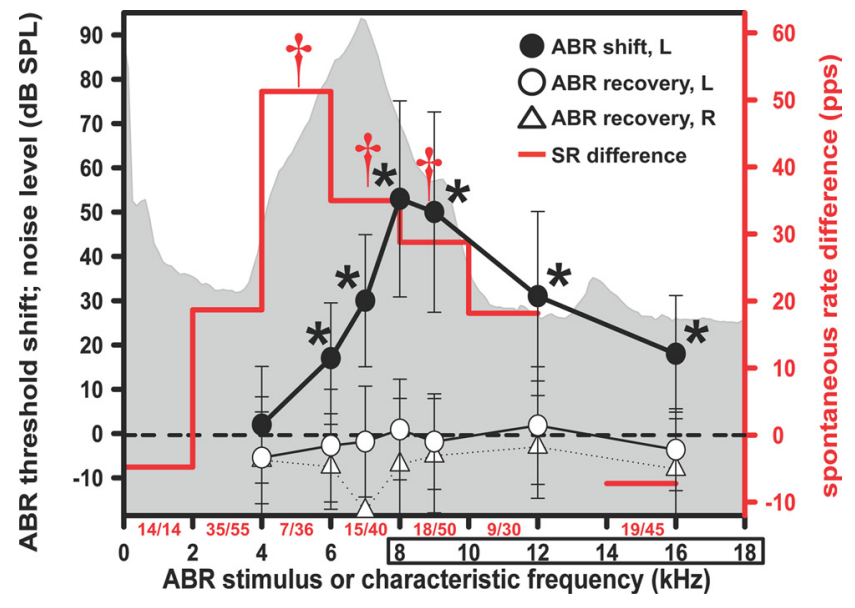

Figure 3. Narrow band noise exposure causes temporary threshold shift but permanently elevated spontaneous rates. The exposure-noise spectrum (gray area) is compared with the immediate threshold shift within an hour after the noise exposure (black symbols; mean and SD of 6 animals/10 $A B R s$ of first and second noise exposures). Due to the considerable length of the noise exposure and $A B R$ recordings, $A B R s$ immediately after the noise exposure could not be recorded for all exposed animals. ABR threshold shift on the day of the DCN recordings (10 - $21 \mathrm{~d}$ after the second noise exposure) has recovered toward $0 \mathrm{~dB}$ (white circles, left exposed ear; white triangles, right ear; 11 animals), while SRs were elevated (red graph; control: 9 animals/135 units; noise-exposed: 12 animals/296 units). Blackstars indicate significant differences for ABRshifts ( $p<0.001$; for detailssee Results), red crosses indicate significance for SR differences ( $p<0.05$, for details see Results). Box around 8-18 kHzlabels the tinnitus frequencies (Fig. 4). Red numbers at the $x$-axis are $N$ for each bin of spontaneous rate differences given for control/noise exposed animals $(n / n)$.

2011). The electrical stimulus at $60 \mu \mathrm{A}$ preceded the tone stimulus by 20 $\mathrm{ms}$ and consisted of 2 biphasic square pulses, $100 \mu$ s per phase, with the negative phase first, $1000 \mathrm{~Hz}$ pulse frequency. The stimulating probe was dipped in Fluoro Gold before insertion. After the experiment current was passed through the stimulation probe and one site of the recording probe to create lesions and to transport the Fluoro Gold into the tissue. The animals were killed and decapitated, and the head was fixed in paraformaldehyde for $4 \mathrm{~d}$. The brain was removed from the skull, fixed overnight, immersed in sucrose solution and then cryosectioned $(100 \mu \mathrm{m}$ thickness). Slices were stained with neutral red and the lesions and Fluoro Gold marks observed with light and fluorescent microscopy.

Data analysis. Waveforms were sorted using principle component and cluster analysis of the waveform shape (OpenSorter, Tucker-Davis Tech- 
A Gap-PPI: normalized startle

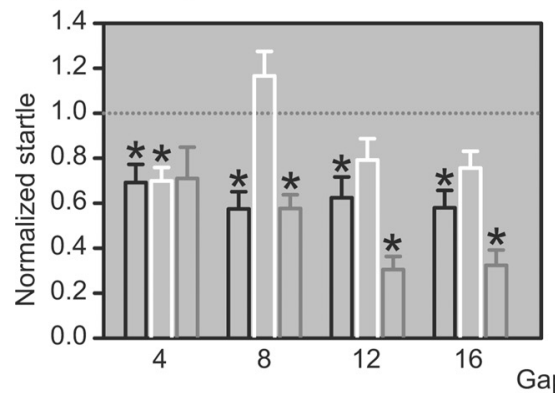

B Gap-PPI: absolute startle

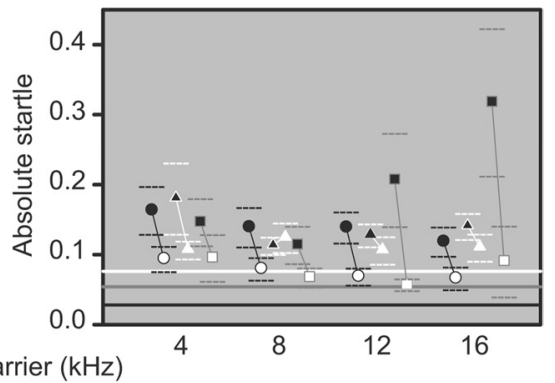

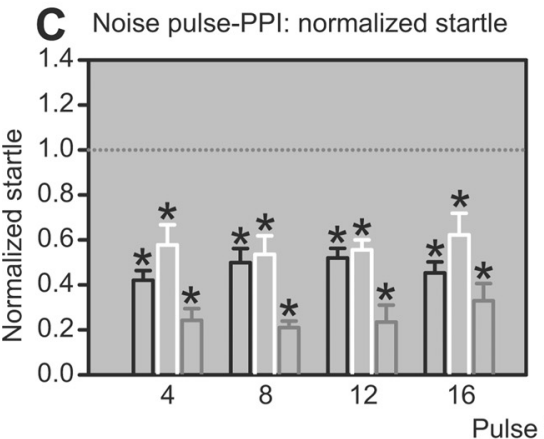

D Noise pulse-PPI: absolute startle
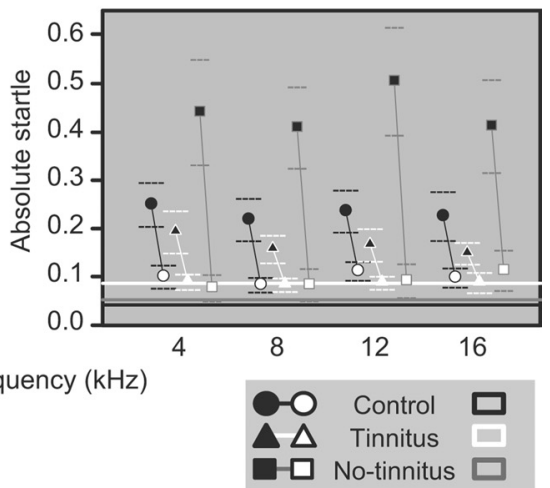

Figure 4. Narrow band noise exposure results in tinnitus in the $8-18 \mathrm{kHz}$ band. Animals with noise exposure were divided into two groups: those with no significant gap detection in gap-carrier bands $8-18 \mathrm{kHz}$ - "tinnitus group," and those with significant gap detection at all carrier bands - "no-tinnitus group." $\boldsymbol{A}, \boldsymbol{B}$, Gap-PPI; C, D, noise pulse-PPI. A, C, The normalized startle (startle with the gap or noise pulse/startle without the gap or noise pulse) is shown for the two noise-exposure groups and the control group after the noise/sham exposure. The dotted line designates a normalized startle of 1, i.e., the startle amplitude without gap or noise pulse. $\boldsymbol{B}, \boldsymbol{D}$, Dot plot of the absolute startle without the gap (black symbols) and with the gap (white symbols). The mean and $95 \%$ confidence intervals are shown as dotted horizontal lines below and above the mean. The mean $+95 \%$ confidence interval of the noise-floor is given for each group as a line plot (black: control, white: tinnitus, gray: no-tinnitus). Black bars, circles: sham exposure control group ( $n=4$ animals), white bars, triangles: tinnitus group ( $n=4$ animals), gray bars, squares: $n$ o-tinnitus group ( $n=3$ animals). Normalized startle responses were derived from the mean of all trials over $4 \mathrm{~d}$ of one animal with the gap normalized to the mean of all trials of one animal without the gap. Stars mark significance in two-way repeated-measures ANOVA $(p<0.05$, for details, see Results).

\section{Control Noise-exposed}

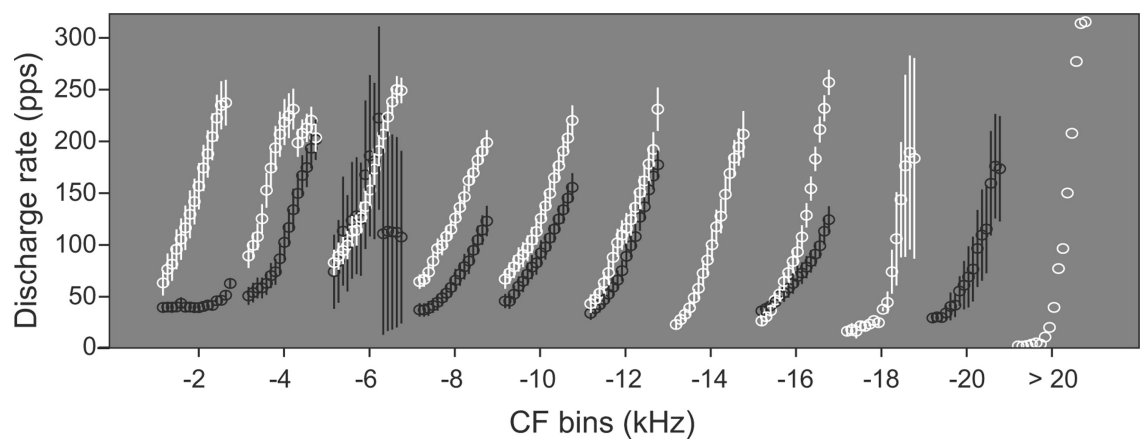

Figure 5. Suprathreshold discharge rates remain elevated after recovery from temporary threshold shift. RLFs of noise exposed animals are steeper and show higher discharge rates across levels. Mean and SE of rate level functions ( $10-85 \mathrm{~dB}$, in $5 \mathrm{~dB}$ steps) are shown within $2 \mathrm{kHz}$ bins of the characteristic frequency of the unit, included are RLFs recorded below/at/above the $\mathrm{CF}$ of the unit. White symbols, noise-exposed animals ( $n=11$ animals/463 RLFs); black symbols, control animals ( $n=9$ animals/252 RLFs).

nologies). K-means clustering with a fixed variance (95\%) and maximum of 5 clusters was used. Data from the respective recording sites was excluded if the shape and size of each cluster and average waveform across the recordings were not consistent. Responses were analyzed for the time window of the tone presentation. Electrical artifacts and ringing that occurred after the current pulses were not included in this time window.

To quantify the immediate and long-term effects of bimodal stimulation the mean rate differences across tone levels were calculated A) (1) between a tone RLF and an immediately subsequent bimodal RLF ("immediate" effect of bimodal stimulation) and (2) between the first tone RLF and a second tone RLF on average $35 \mathrm{~min}$ after the bimodal stimulation ("long-term"). Data points (number of units * sound levels of RLFs) were grouped into rate-enhancement (white graphs; first tone response $<$ bimodal response (1) or first tone response $<$ later tone response (2)) and rate-suppression (black graphs; first tone response $>$ bimodal response (1) or first tone response $>$ later tone response (2)). The data were collapsed across tone level to form pie charts showing the number of data points with enhancement or suppression and the sum of rate changes of all data points (magnitude of rate change summed across units and all sound levels). Arrows and stars in the rate difference plots indicate the results of linear mixed model statistics comparing all observed rate differences of a group (either immediate or long-term effects of animals in the control, noise-exposed, tinnitus or no-tinnitus groups). Each unit is treated as a subject with two difference-RLFs (immediate and long-term): (1) comparison of noise-exposed (or tinnitus and no-tinnitus) versus control animals' data for within immediate rate differences or long-term rate differences. Random intercept for each unit, repeated factor is "sound level of the RLF." (2) comparison of immediate versus longterm rate differences of control animals' data, noise-exposed animals' data, tinnitus animals' data or no-tinnitus animals' data. Random intercept for each unit, repeated factor is "immediate and long-term" and "sound level of the RLF." Pairwise comparison of immediate versus long-term or control versus exposed, tinnitus or no-tinnitus was done across all sound levels (significances labeled with stars), adjustment for multiple comparisons: Sidak, critical level $p<0.05$.

Data analysis, statistics and plotting were performed with OpenExplorer (Tucker-Davis Technologies), SPSS (Version 17), and SigmaPlot (Version 11.0, Systat Software Inc.).

\section{Results}

\section{Narrow band noise exposure causes} temporary threshold shifts, which are accompanied by tinnitus in $\sim 50 \%$ of animals

Overexposure with a narrow band noise centered at $7 \mathrm{kHz}$ resulted in an immediate ABR threshold shift between 6 and 16 $\mathrm{kHz}$ with a maximum of $50 \mathrm{~dB}$ mean threshold shift at $8 \mathrm{kHz}$ (Fig. 3; mean of individual animal's ABR differences before versus immediately after the exposure; nondirectional onesample $t$ test against a mean threshold shift of $0 \mathrm{~dB}$ for each test frequency, $\mathrm{df}=9$, minimum $p<0.001$, maximum $p=0.002$ ). 
Four of the seven animals developed tinnitus for frequencies between 8 and 18 $\mathrm{kHz}$ by the time of recovery of $\mathrm{ABR}$ thresholds from the second exposure (Fig. 4). The normalized startles of these "tinnitus" animals were increased toward or above one; and were maximal in the $8-10$ $\mathrm{kHz}$ band. The "no-tinnitus" animals had decreased normalized startles in the gap and noise pulse test. Stars in Figure 4 mark significance in two-way repeatedmeasures ANOVA on ranks within one frequency of absolute startle data (with gap or noise pulse versus without gap or noise pulse, one mean value across trials of $4 \mathrm{~d}$ per animal). Factors are group ("control, tinnitus, no-tinnitus") and trial (repeated within-subject factor "gap or no gap" and "prepulse or no prepulse"), post hoc all pairwise multiple comparisons with Holm-Sidak, $\mathrm{df}=1$, minimum $p<$ 0.001 , maximum $p=0.04$.

\section{Narrow band noise exposure produces temporary threshold ABR shifts but long-term elevations of spontaneous and tone-evoked DCN unit discharge rates}

Although the ABR thresholds had recovered by the time the DCN recordings were made (average $16 \mathrm{~d}$ after the second exposure, $10-21 \mathrm{~d}$, mean of individual animal's ABR differences before versus at the day of DCN recording; nondirectional one-sample $t$ test against a mean threshold shift of $0 \mathrm{~dB}$ for each test frequency, $\mathrm{df}=10$, minimum $p=0.111$, maximum $p=0.796$ ), spontaneous rates (SRs) were significantly elevated in noise-exposed animals compared with control animals for units with CFs between 4 and $10 \mathrm{kHz}$ (Fig. 3). Pairwise comparisons were made between the SRs of noise-exposed and control animals with ANOVA (Fig. 3, red crosses), between-subject effects "group noise-exposed or control" and "CF-bin" and interaction between both, adjustment for multiple pairwise comparisons within 9 CF bins: Holm-Sidak, corrected total $\mathrm{df}=430$, for CFs $4-10 \mathrm{kHz}$ : minimum $p=0.029$, maximum $p=0.039$.

Moreover, suprathreshold discharge rates were elevated for tone levels up to $85 \mathrm{~dB}$ and CFs up to $16 \mathrm{kHz}$ (Fig. 5). This resulted in steeper RLFs for the noise-exposed animals especially for tones above CF (Fig. 6). The difference was significant at all levels above $\mathrm{CF}$, between 45 up to $85 \mathrm{~dB}$ at CF and 55 up to $85 \mathrm{~dB}$ below CF (ANOVA, between-subject effects "noise-exposed or control" and "dB level" and interaction between both, adjustment for multiple pairwise comparisons at $18 \mathrm{~dB}$ levels: HolmSidak; above CF: corrected total $\mathrm{df}=5695$, for $0-85 \mathrm{~dB}$ : minimum $p=0.000$, maximum $p=0.003$; at CF: corrected total $\mathrm{df}=2843$, for $45-85 \mathrm{~dB}$ : minimum $p=0.001$, maximum $p=$ 0.039; below $\mathrm{CF}$ : corrected total $\mathrm{df}=4239$, for $55-85 \mathrm{~dB}$ : minimum $p=0.000$, maximum $p=0.033$ ).

\section{Narrow band noise exposure amplifies somatosensory modulation of sound-driven responses and results in dominant long-term enhancement}

To explore changes in auditory-somatosensory integration in the DCN after cochlear damage, rate differences between toneevoked responses before, during and after bimodal stimulation (tri- geminal pulse preceding tone stimulation) were compared for noiseexposed and control animals (top and bottom row, Fig. 7A-C). This comparison was done for the immediate and long-term rate differences (left and right column charts, Fig. $7 A-C$ ).

The immediate mean enhancement and suppression are larger after noise exposure (Fig. 7A, left column graphs). This, together with the larger number of data points with enhancement in the noise-exposed animals compared with the control animals (Fig. $7 B$, left column pie charts), results in a greater bimodal enhancement in the noise-exposed group compared with the control animals (Fig. 7C, left column pie charts). Comparing the immediate and long-term rate differences reveals a further increase in mean enhancement in the noise-exposed animals and in contrast, an increase in enhancement and especially suppression for the control animals (Fig. 7A, compare left versus right column graphs). At the same time the number of data points with longterm enhancement is only slightly smaller than the number of data points for the immediate rate differences in the noiseexposed animals (Fig. $7 B$, top row pie charts) while the number of data points showing suppression increases in the control animals (Fig. $7 B$, bottom row pie charts). This opposite shift of enhancement and suppression between the noise-exposed and control animals from the immediate to the long-term effects results in a dominant long-term enhancement in noise-exposed animals, contrasting with a dominant long-term suppression in control animals (Fig. 7C, right column; Fig. 7A: significant difference of the long-term effect between noise-exposed vs control indicated with arrow and star; linear mixed model statistics, details see methods/data analysis, adjustment for pairwise multiple comparisons: Sidak, $\mathrm{df}=140.942, p=0.000)$.

\section{Tinnitus correlates with dominant long-term enhancement by somatosensory inputs}

To assess the role of somatosensory-auditory integration in tinnitus, comparisons were made between the control animals and 
A
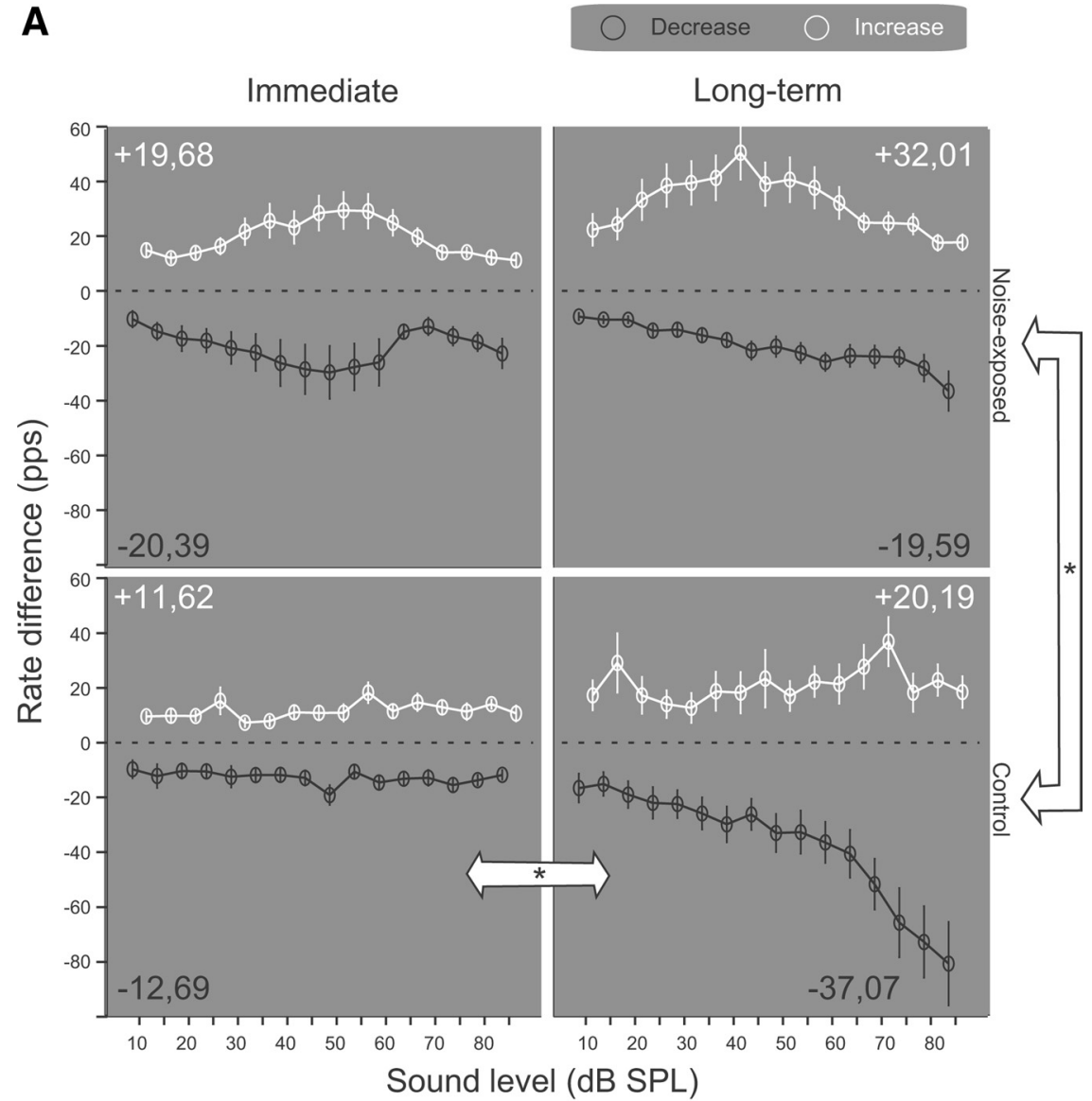

\section{B Number of data points}

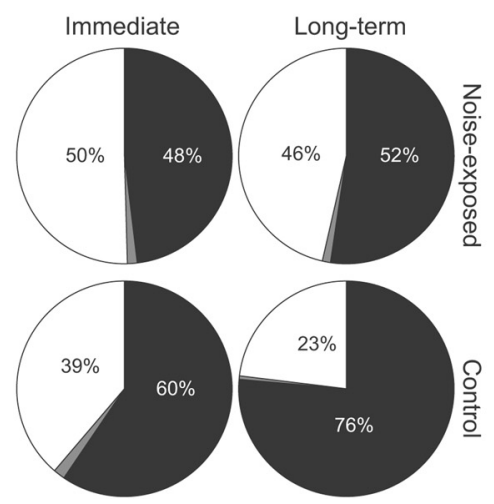

C Sum of changes

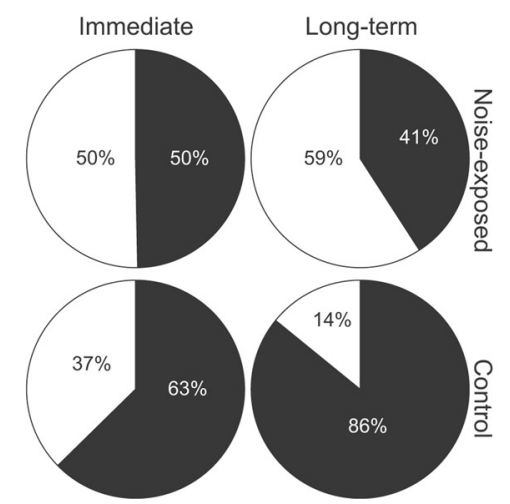

Figure 7. Effect of noise exposure on the balance between bimodal enhancement and suppression. $\boldsymbol{A}$, Left, Immediate rate differences between tone RLFs and immediately following bimodal RLFs (control: $n=8$ animals/50 RLFs, noise-exposed: $n=11$ animals/108 RLFs). All RLFs were recorded at the units' (F. Right, Long-term rate differences between the first tone RLF and a second tone RLF recorded later in time after bimodal stimulation (control, $n=8$ animals/49 RLFs; noise-exposed, $n=10$ animals/93 RLFs). The mean and SE are shown for suppression i.e., reduction of the discharge rate (black dots) and enhancement i.e., increase of discharge rate (white dots). The mean across level is given as a black or white number in the graph. Noise-exposed and control animals are shown in the top and bottom, respectively. Star and arrow marks significant difference (linear mixed model statistics, adjustment for multiple comparisons: Sidak, $p<0.05$; for details see Materials and Methods, Data analysis). Data of $\boldsymbol{A}$ are collapsed across sound levels as pie charts in $\boldsymbol{B}$ and $\boldsymbol{C}$. $\boldsymbol{B}$, The number of data points with enhancement (white slices) or suppression (black slices) or no change (gray slices). $C$, The sum of immediate and long-term rate differences (sum of rate difference from all units at all levels from 10 to $85 \mathrm{~dB}$ ) for noise-exposed and control animals. Colors as in $\boldsymbol{B}$.

noise-exposed animals that developed tinnitus or did not develop tinnitus (Fig. 8). The mean immediate rate differences for the tinnitus group were the same as those of the control animals (Fig. $8 A$, left column), whereas the no-tinnitus group showed larger enhancement as well as larger suppression exceeding the effect of the noise exposure shown in Figure $7 A$. The group of tinnitus animals stands out in terms of its longterm bimodal effect: comparing the immediate and long-term rate differences reveals an increase in enhancement over time in the tinnitus group (Fig. $8 \mathrm{~A}$, bottom row). This results in a significant difference between the immediate and long-term data for the tinnitus group across sound levels [Fig. $8 A$; significance of linear mixed model statistics indicated with arrow and star (for details see Materials and Methods, Data analysis); adjustment for multiple pairwise comparisons: Sidak, $\mathrm{df}=943.340, p=0.000]$. This shift from the immediate to the long-term rate differences raises the mean rate enhancement for the tinnitus group above the level of enhancement for the control animals, resulting in a significant difference between the animals with tinnitus and the control animals of the long-term rate differences across level (Fig. 8A; compare bottom with middle row graphs; significance of linear mixed model statistics indicated with arrow and star, adjustment for multiple pairwise comparisons: Sidak, $\mathrm{df}=104.295, p=0.000)$. The increase in mean enhancement (Fig. $8 A$, bottom row) together with an increase in the number of data points with enhancement (Fig. $8 \mathrm{~B}$, bottom row pie charts) results in a dominant long-term enhancement in the tinnitus group (Fig. $8 \mathrm{C}$, bottom pie charts). In contrast, the no-tinnitus group is characterized by a decrease in the mean suppression and smaller increase in the mean enhancement [Fig. $8 A$, top row graphs: significance of linear mixed model statistics is indicated with arrow and star (for details see Materials and Methods, Data analysis); adjustment for multiple pairwise comparisons: Sidak, df $=1186.949, p=0.000]$. The control group shows an increase in suppression and enhancement (Fig. 8A, middle row graphs). Moreover, the control animals and no-tinnitus animals show a decreased number of data points with enhancement and an increased number of data points with suppression (Fig. $8 \mathrm{~B}$ top and middle row pie charts) contrasting with the group of tinnitus animals, which shows an increased number of data points with rate enhancement. Together, these effects result in a clear dominance of long-term somatosensory (bimodal) enhancement in the animals with tinnitus in contrast to control animals and exposed no-tinnitus animals (Fig. $8 \mathrm{~B}$; significant differences between the animal groups' long-term rate differences in linear mixed model statistics indicated with arrows and stars, details see 
methods/data analysis, adjustment for multiple pairwise comparisons: Sidak, tinnitus vs control: $\mathrm{df}=104.295, p=$ 0.000; tinnitus vs no-tinnitus: $\mathrm{df}=$ 104.295, $p=0.036)$.

\section{Buildup and chopper units show differential changes after noise exposure and tinnitus}

Units were categorized based on the temporal response patterns at different sound levels. The two major response types were buildup and chopper (Fig. 9). In the tinnitus group, buildup units' spontaneous and tone-evoked rates were increased compared with control animals and notinnitus animals (Figs. 10, 11). The increase was significant between 25 and 85 dB (Fig. 11, ANOVA, between-subject effects "tinnitus, no-tinnitus, control" and "dB level" and interaction between both, adjustment for multiple pairwise comparisons at $18 \mathrm{~dB}$ levels: Sidak, corrected total $\mathrm{df}=596$, control vs tinnitus, 25-85 dB: minimum $p=0.000$, maximum $p=0.044$ ). This rate increase affected the onset and buildup portion of the response and prolonged the response-duration (Fig. 11B). Buildup units of the no-tinnitus animals were not different from the control group. In contrast to buildup units, chopper units of tinnitus and no-tinnitus animals did not show increased SR (Fig. 10, ANOVA, between-subject effects "tinnitus, notinnitus, control" and "response type" and interaction between both, adjustment for pairwise multiple comparisons: Sidak, corrected total $\mathrm{df}=112$; buildup control vs buildup tinnitus: $p=0.026$; buildup no-tinnitus vs buildup tinnitus: $p=$ 0.005). In chopper units the increase of sound-evoked rates in no-tinnitus animals were significantly different from the control animals only at 40 and $45 \mathrm{~dB}$ (Fig. 12, ANOVA, between-subject effects "tinnitus, no-tinnitus, control" and " $\mathrm{dB}$ level" and interaction between both, adjustment for multiple pairwise comparisons at $18 \mathrm{~dB}$ levels: Sidak, corrected total $\mathrm{df}=1025$; control vs no-tinnitus, $40 \mathrm{~dB}: p=0.045,45 \mathrm{~dB}: p=0.035)$.

In the tinnitus group, the long-term somatosensory (bimodal) influence on buildup units as well as chopper units (Figs. 13, 14) was predominantly enhancing. Buildup units of the control and notinnitus animals showed predominantly suppressive long-term effects (Fig. 13). Chopper units in control animals showed predominantly suppressive long-term effects, whereas the chopper units in no-tinnitus animals showed more balanced enhancement and suppression (Fig. 14).

Although the low number of units did not allow a statistical analysis within single response types and behavioral/noise ex-

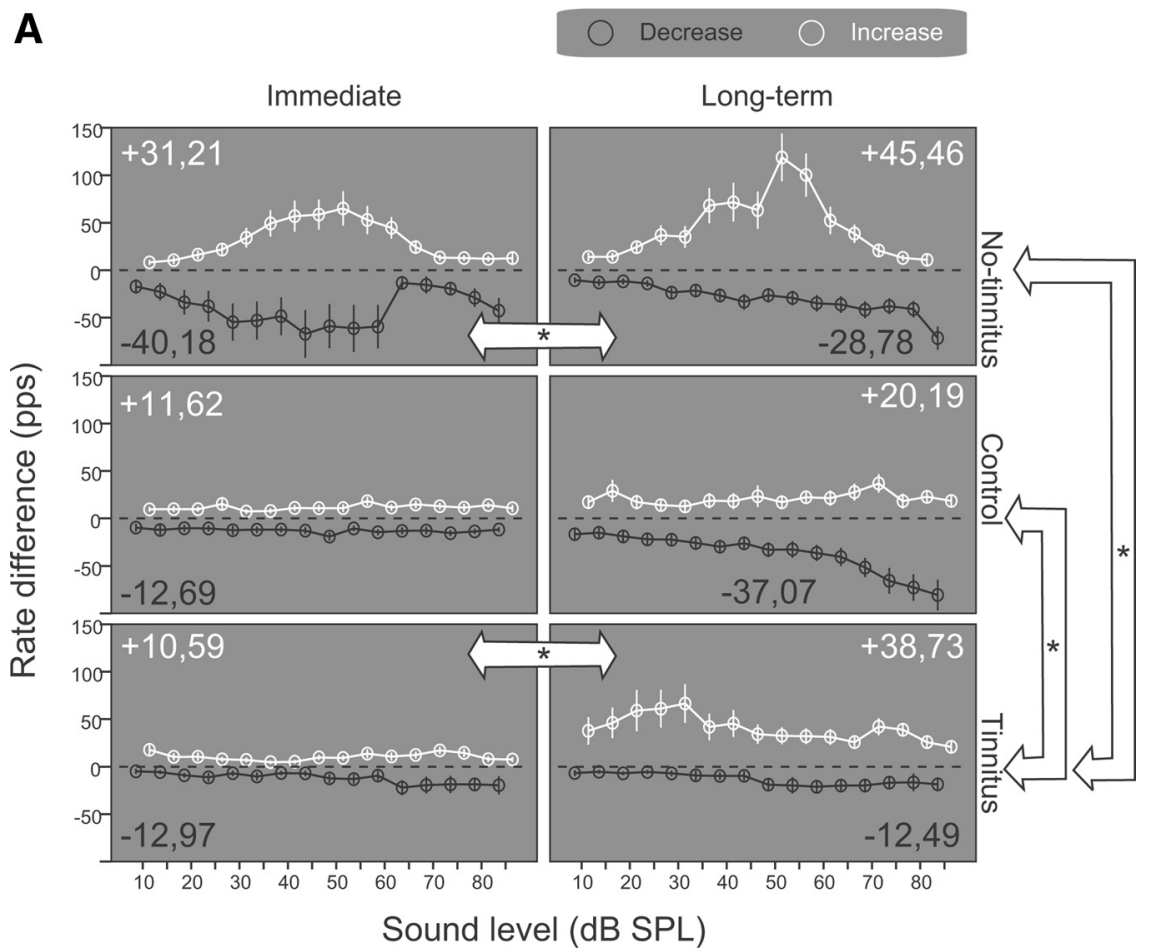

B Number of data points

C Sum of changes
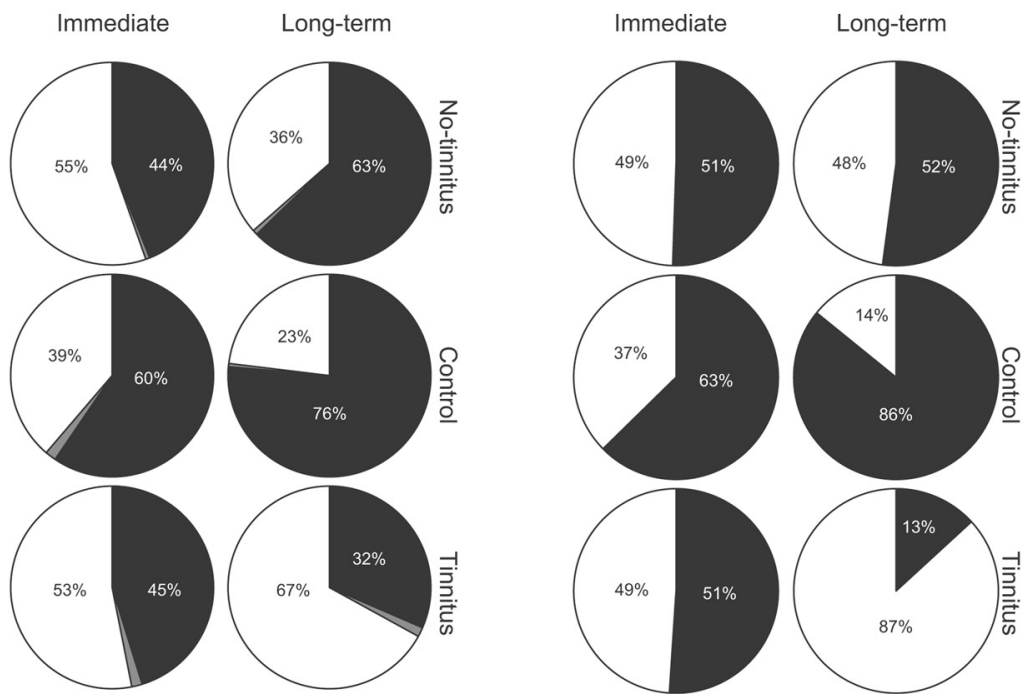

Figure 8. Tinnitus is accompanied by a strengthening of bimodal enhancement. $A$, Rate differences (1) between RLFs during unimodal tone stimulation and immediately following RLFs during bimodal tone and somatosensory stimulation ("immediate" change): control, $n=8$ animals/50 RLFs; no-tinnitus, $n=3$ animals/39 RLFs; tinnitus, $n=4$ animals/30 RLFs and (2) between the first unimodal tone RLFs and a second tone RLFs repeated later in time (Long-term): control, $n=8$ animals/49 RLFs; no-tinnitus, $n=3$ animals/33 RLFs; tinnitus, 3 animals/25 RLFs. All RLFs were recorded at the units' CF. The pie charts show the number of data points ( $\boldsymbol{B}$ ) and the summed immediate and long-term rate difference of the data ( $\boldsymbol{C}$. Compared are control animals with noiseexposed animals that developed tinnitus or with noise-exposed animals that did not develop tinnitus. Stars and arrows mark significant differences (linear mixed model statistics, adjustment for multiple comparisons: Sidak, $p<0.05$; see Materials and Methods, Data analysis). For a detailed description see Figure 7 legend.

posure groups, the long-term somatosensory effects of buildup and chopper units (Figs. 13, 14) were similar to each other. In both response types somatosensory (bimodal) enhancement predominated in animals with tinnitus. This similarity exists despite the different behavior of the tone and spontaneous responses, which are only increased in buildup units (Figs. 10-12). 

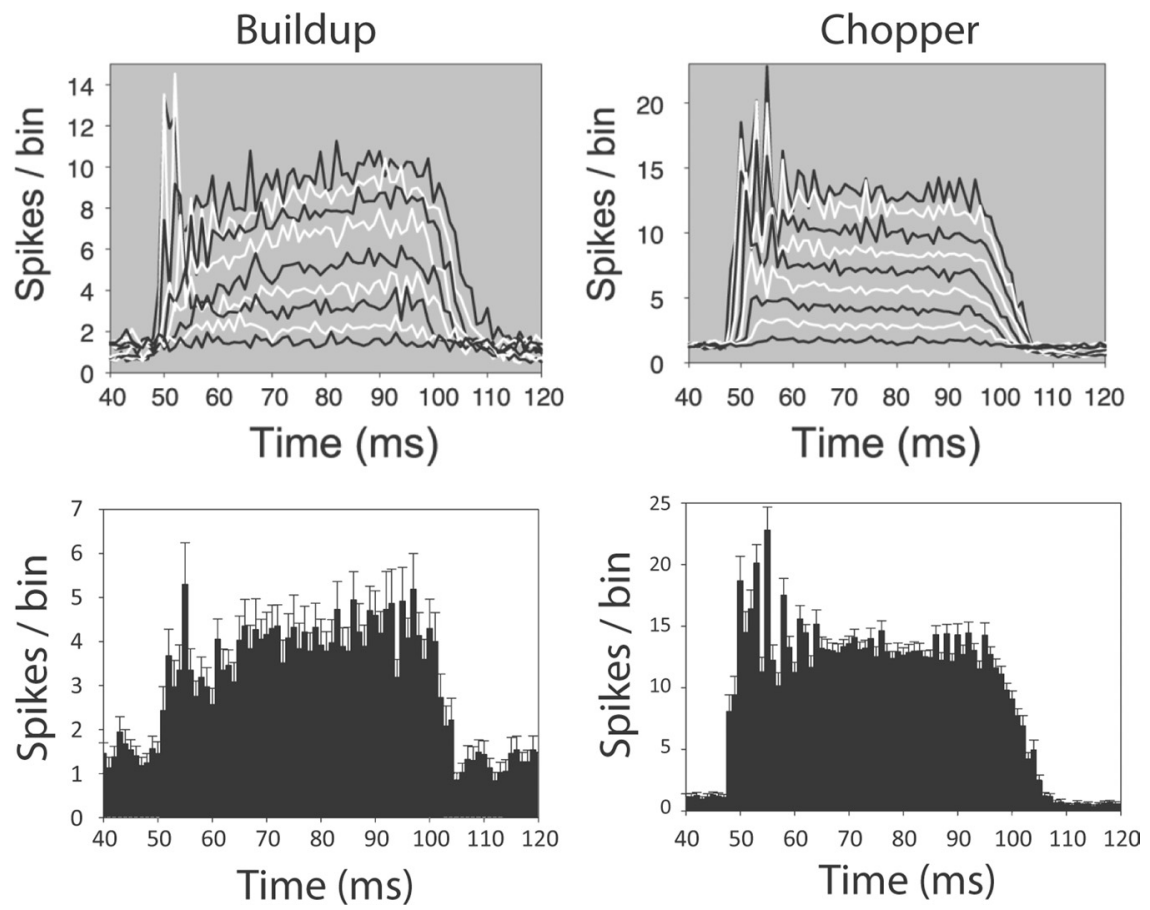

Figure 9. Predominant temporal response types were buildup and chopper. Poststimulus time histograms (PSTHs) were determined from RLFs at the unit CF. Population (mean) PSTHs of the two main response types buildup (left) and chopper (right) are shown in the top panels. Levels between $10 \mathrm{~dB}$ and $90 \mathrm{~dB}$ are indicated with alternating black and white line plots. Bottom shows single level group PSTHs (mean and SD) at $35 \mathrm{~dB}$ for buildup and $85 \mathrm{~dB}$ for chopper. The buildup response was most characteristic at lower levels and accompanied by onset components at higher levels (left). The precision of the chopping response increased with level (right).

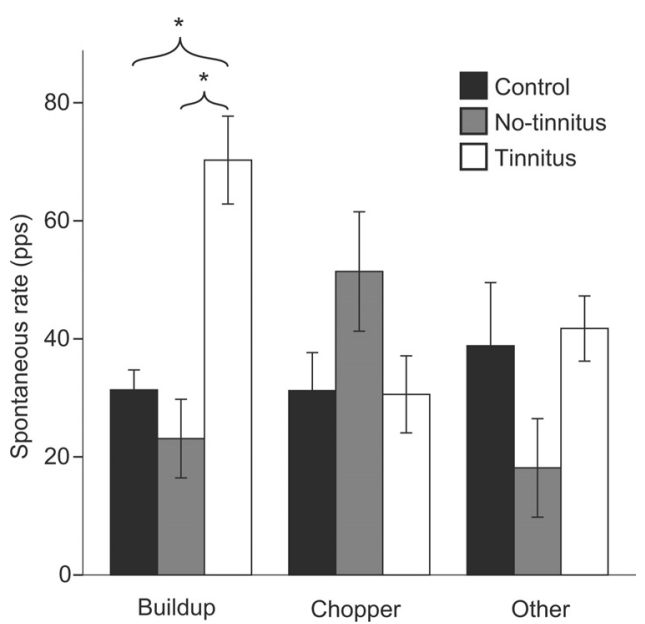

Figure 10. Spontaneous rates are increased in buildup units of animals with tinnitus. Mean and SE of SRs of control animals (black), noise-exposed tinnitus animals (white) and no-tinnitus animals (gray) are plotted for the main response types. Stars indicate significant differences in ANOVA ( $p<0.05$, adjustment for multiple comparisons: Sidak; for details, see Results). Buildup control: $n=5$ animals/ 12 units, buildup no-tinnitus: $n=2$ animals/ 12 units, buildup tinnitus: $n=2$ animals/ 7 units, chopper control: $n=5$ animals/23 units, chopper no-tinnitus: $n=2$ animals/17 units, chopper tinnitus: $n=3$ animals/15 units, other control: $n=6$ animals/16 units, other no-tinnitus: $n=3$ animals/ 3 units, other tinnitus: $n=3$ animals $/ 8$ units.

\section{Discussion}

This study is the first to investigate long-term effects of somatosensory-auditory processing in vivo and its plasticity after temporary noise-induced threshold shift. Long-term somatosensory effects shifted from dominant suppression in normal animals to enhancement in animals with tinnitus. This shift might explain the close connection between tinnitus and the somatosensory system and highlights the importance of incorporating the somatosensory system into tinnitus treatment strategies.

\section{Tinnitus is correlated with ABR threshold shifts}

Narrow band noise exposure resulted in behavioral evidence of tinnitus in the $8-18 \mathrm{kHz}$ bands with a maximum effect at $8-10 \mathrm{kHz}$. This correlates well with the TTS between 6 and $16 \mathrm{kHz}$ with a maximum at 8 and $9 \mathrm{kHz}$ and is consistent with studies showing the tinnitus spectrum overlapping the hearing loss region (Lockwood et al., 2002; Noreña et al., 2002; Eggermont and Roberts, 2004; Roberts et al., 2006; Schaette and Kempter, 2009). The maximum TTS shifted $0.2-0.3$ octaves extending to frequencies $>10$ $\mathrm{kHz}$. Elevated ABRs at and above the exposure frequency are commonly observed after acoustic trauma (Brozoski et al., 2002; Kujawa and Liberman, 2009; Mulders et al., 2011; Vogler et al., 2011).

\section{SR elevations occur at frequencies below the tinnitus and ABR threshold shifts}

The present study is the first to correlate the frequency profile of elevated SR with the tinnitus frequency after TTS. Although increased SRs resided within the noise exposure band it was skewed to frequencies below the ABR threshold elevations. This agrees with shifts in the noise-induced SR elevation of DCN neurons over time from frequencies below the exposure frequency to higher frequency regions (Kaltenbach et al., 2000). Our data show that tinnitus resides in bands at and above the maximum TTS and argues against the view that neurons with elevated SRs reside precisely in CF regions of the tinnitus pitch (Kaltenbach et al., 2004).

\section{Suprathreshold discharge rates remain elevated after ABR} recovery and may reflect hyperacusis and/or tinnitus

Although ABRs had recovered by the time of neural recordings, DCN responses showed persistent increases in SRs and steeper tone RLFs across the DCN unit population. The increased spontaneous and suprathreshold firing rates were observed in buildup units in tinnitus animals. This corroborates previous results for pauser buildup and wide chopper units (Brozoski et al., 2002). In contrast, the chopper type in our study, which is comparable to choppers shown in (Stabler et al., 1996), did not show increased spontaneous and suprathreshold rates. In contrast to the steeper RLFs shown here after TTS, ANF RLFs were shallower after TTS (Kujawa and Liberman, 2009). While the shallower ANF slopes may be due to deficient hair cell-ANF synapses, the steeper DCN RLFs likely reflect central plasticity to counteract the reduced ANF inputs (Cai et al., 2009; Schaette and Kempter, 2009; Norena, 2011). Downregulation of inhibitory inputs accompanying tinnitus after auditory insults (Wang et al., 2009; Middleton et al., 2011) would further counteract the reduced ANF input; 
reduction in sideband inhibition could explain the larger increase in suprathreshold response-rates above CF found in our study.

The increase in tone-evoked responses across frequency regions after TTS might point to hyperacusis, which in humans affects all frequencies (Anari et al., 1999; Schaaf et al., 2003). Hyperacusis would likely result in decreased normalized startles as the gap carrier and noise-pulse are both well above hearing threshold. In addition it would increase the absolute startle in the absence of a preceding gap/noise pulse (Sun et al., 2009) as demonstrated by the no-tinnitus group in our study (Fig. 4). Tinnitus and hyperacusis often cooccur (Anari et al., 1999; Schaaf et al., 2003; Nelson and Chen, 2004; Dauman and Bouscau-Faure, 2005). If cooccurring in the no-tinnitus group, their effects on normalized startle responses in the gap-PPI test could counteract each other, with tinnitus increasing and hyperacusis decreasing the normalized startle.

\section{Short and long-term effects of bimodal stimulation}

Whereas the immediate effects of trigeminal and dorsal column stimulation have been described previously (Shore, 2005; Shore et al., 2007; Kanold et al., 2011; Koehler et al., 2011) long-term effects have not been studied in vivo. In the present study, bimodal enhancement or suppression of sound-driven responses was investigated $\sim 35 \mathrm{~min}$ after repeated auditory-somatosensory stimulation. This timeframe is similar to that of long-term depression and potentiation (LTD/LTP) at the parallel fiber-cartwheel/fusiform cell synapses in vitro (Fujino and Oertel, 2003; Tzounopoulos et al., 2004, 2007; Zhao et al., 2011). Although LTP/LTD was not shown at the auditory nerve-fusiform synapses (Fujino and Oertel, 2003; Zhao et al., 2011), several mechanisms could explain the long-term bimodal effects on auditory responses as consequences of long-term synaptic plasticity at the parallel fiber synapses. Assuming that spontaneously active parallel fibers regulate fusiform and cartwheel cell excitability, long-term plasticity at the parallel fiber synapses would modify the excitability of the cells and hence change their auditory responses. Moreover, changes in postsynaptic excitability as consequences or coeffects of LTP/LTD and the spread of LTP/LTD to neighboring synapses (Dan and Poo, 2006; Doiron et al., 2011) might also result in the long-term bimodal effects seen in our study.

In cerebellar-like circuits LTP/LTD modifies parallel fiber input, thereby can-
A

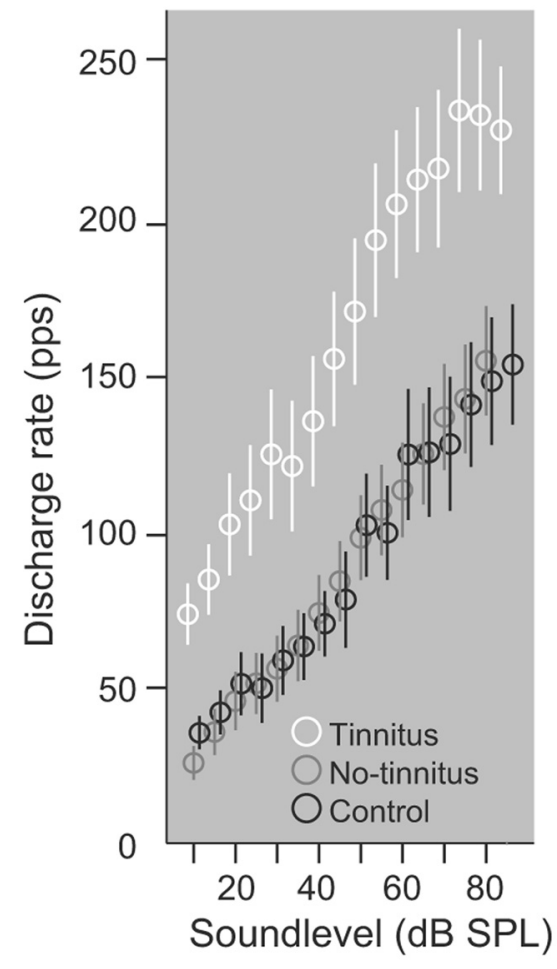

B

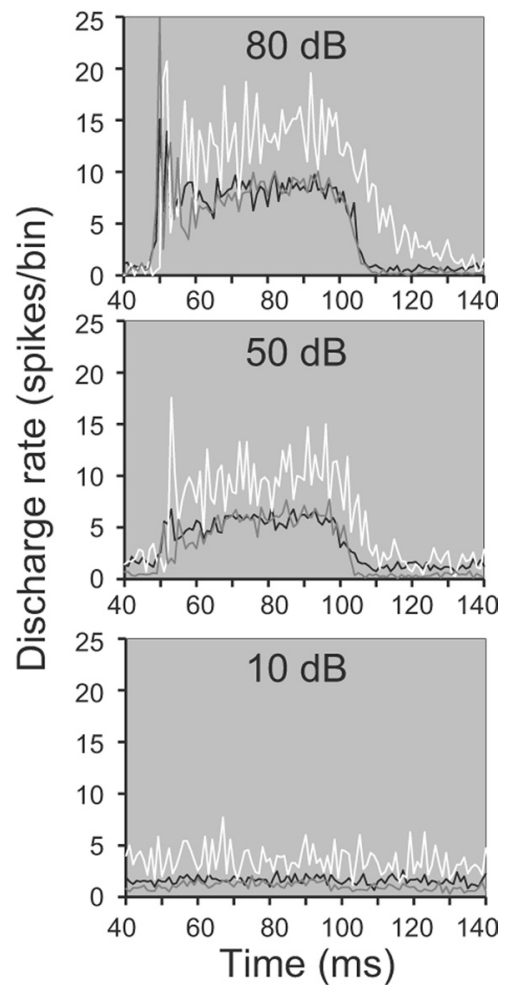

Figure 11. Buildup units in tinnitus animals show elevated tone responses. $\boldsymbol{A}, \boldsymbol{B}$, Population rate level functions $(\boldsymbol{A})$ and PSTHs $(\boldsymbol{B})$ of buildup units in control animals (black plots) and noise-exposed animals that developed tinnitus (white plots) and those that did not develop tinnitus (gray plots). PSTHs are shown for 10, 50, and $80 \mathrm{~dB}$. Tinnitus, $n=2$ animals/7 units; no-tinnitus, $n=2$ animals/16 units; control, $n=5$ animals/11 units.
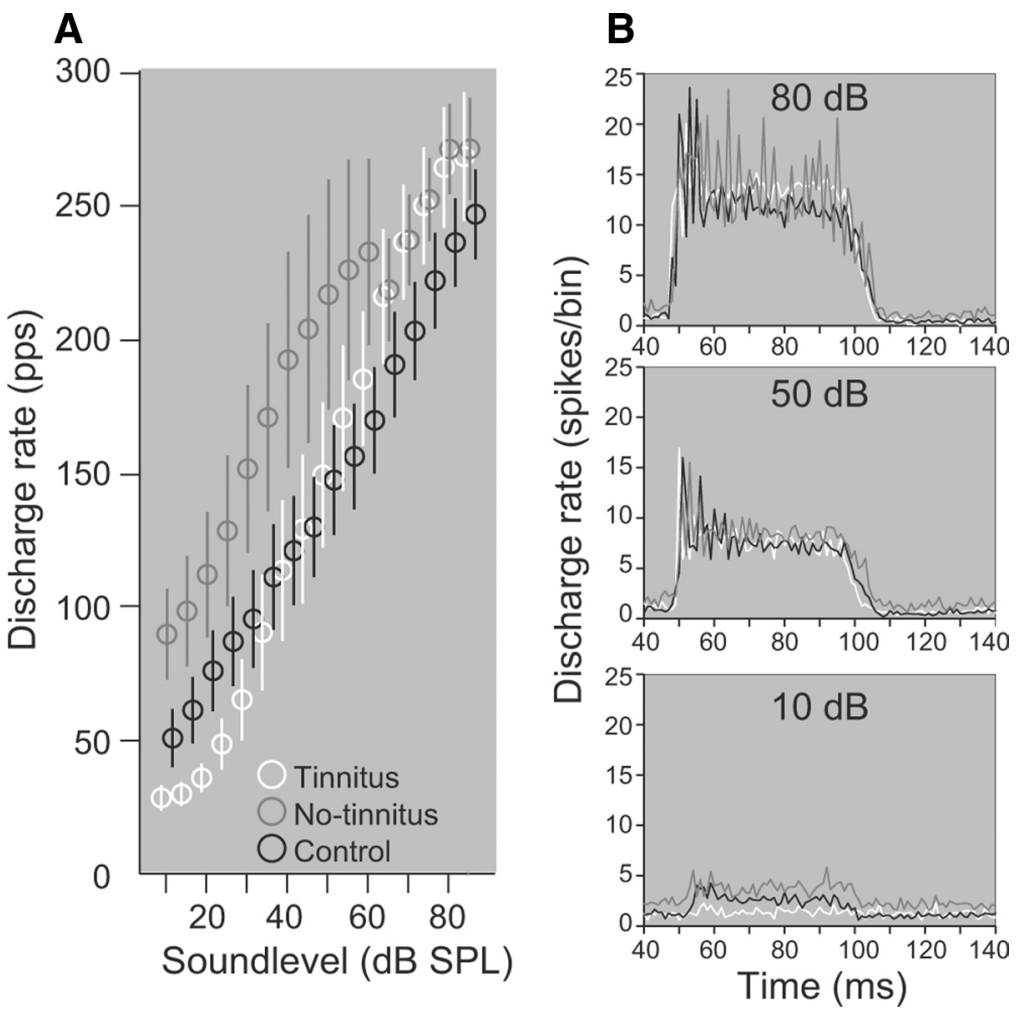

Figure 12. Chopper units in tinnitus animals do not show elevated tone-responses. $\boldsymbol{A}, \boldsymbol{B}$, Population RLFs $(\boldsymbol{A})$ and PSTHs $(\boldsymbol{B})$. For details see legend of Figure 11. The elevation of rates for no-tinnitus animals were not significant. Tinnitus, $n=3$ animals $/ 15$ units; no-tinnitus, $n=2$ animals/ 19 units; control, $n=5$ animals $/ 23$ units. 
A
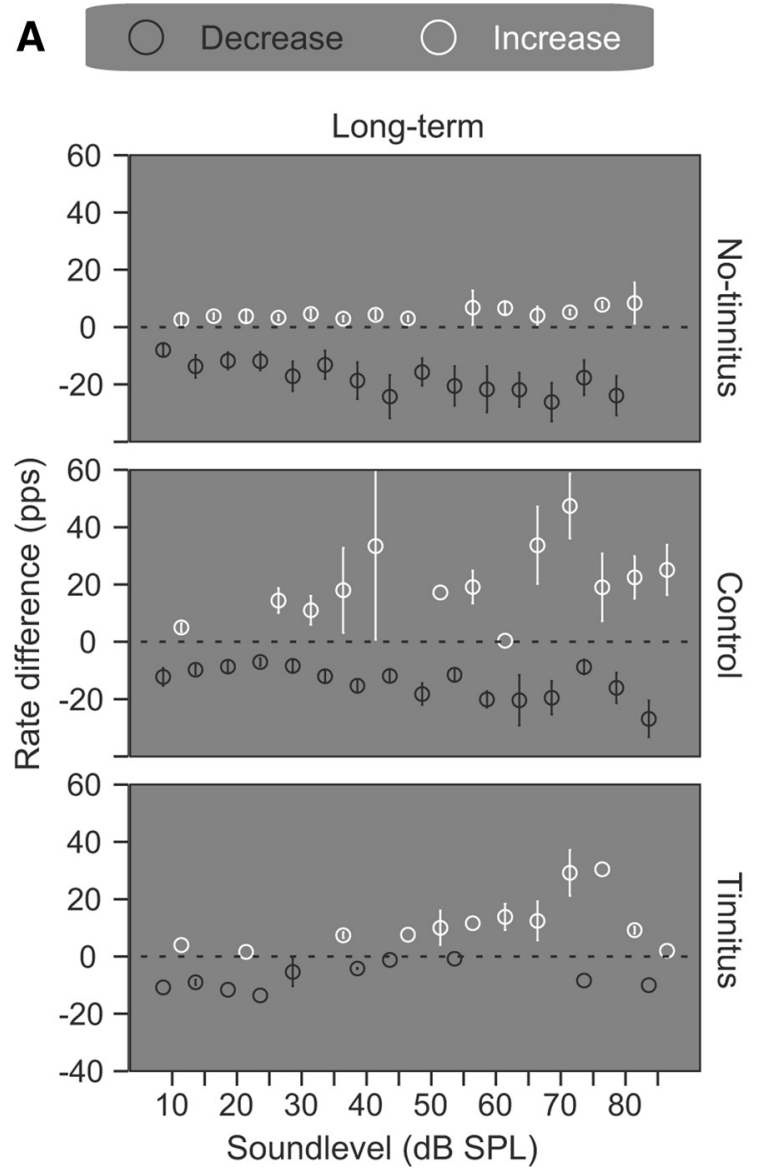
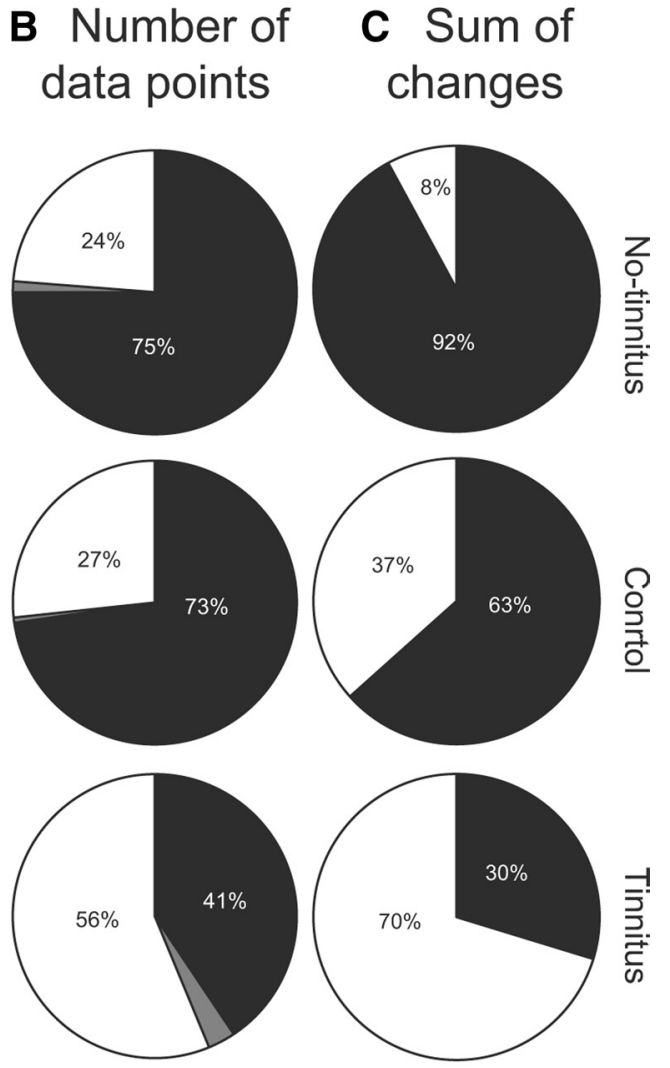

을

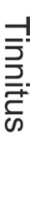

Figure 13. Long-term somatosensory (bimodal) effects in buildup units are predominated by enhancement in tinnitus animals. $A$, Rate differences between the first unimodal tone RLFs and a second tone RLFs repeated later in time: control, $n=5$ animals/11 RLFs; no-tinnitus, $n=2$ animals/16 RLFs; tinnitus, $n=1$ animal/2 RLFs. All RLFs were recorded at the units' CF. $\boldsymbol{B}, \boldsymbol{C}$, The pie charts show the number of data points $(\boldsymbol{B})$ and the summed rate difference $(\boldsymbol{C})$. Control animals are compared with noise-exposed animals that developed tinnitus or with noise-exposed animals that did not develop tinnitus. Suppression, i.e., a reduction of discharge rate, is shown as black plots or pies and enhancement, i.e., increase of discharge rate, as white plots or pies. For a detailed description see legend of Figure 7.

celing expected sensory input (Oertel and Young, 2004; Bell et al., 2008). Plasticity of the parallel fiber input results in a "negative image," which is the inverse of the expected sensory input. In electrosensory systems the negative image can be stored for at least 38 min or until a changing parallel fiber input further modifies the negative image (Bell, 1986, 2001). The capability of cerebellar-like systems to store and retrieve effects of parallel fiber inputs' plasticity to create negative images might underlie the lasting effect of the bimodal stimulation on the acoustically evoked responses. However in vivo studies of synaptic plasticity are necessary to investigate plasticity and memory functions in the DCN.

\section{Strengthened bimodal enhancement counteracts loss of auditory input and accompanies tinnitus}

In contrast to control animals, which showed predominant bimodal suppression, long-term bimodal enhancement predominated after noise exposure, was especially prominent in tinnitus animals, and was observed in chopper as well as buildup units. Predominant bimodal suppression in control animals is consistent with one proposed function of the somatosensory inputs, to suppress responses to self-generated inputs (Bell et al., 1997). The predominant bimodal enhancement after noise exposure points to plastic changes affecting somatosensory inputs to the DCN, which might result in tinnitus development: Strengthening of excitatory, somatosensory inputs or increasing the sensitivity to those inputs could reflect homeostatic plasticity of multisensory DCN neurons restoring normal discharge activity (Kaltenbach et al., 2005; Schaette and Kempter, 2009; Zeng et al., 2009; Norena, 2011) after reduction of ANF input (Kujawa and Liberman, 2009). The differential expression of distinct vesicular glutamate transporters (VGLUTs) in ANF and somatosensory terminals allows for examining this theory. Whereas ANF synapses in the deep layer of DCN express VGLUT1, non-auditory, glutamatergic inputs from cuneate and Sp5 nuclei in the granule cell domain express VGLUT2 (Zhou et al., 2007; Zeng et al., 2009, 2011). Two weeks after unilateral cochlear damage, VGLUT2 immunoreactivity was upregulated and VGLUT1 downregulated in the ipsilateral cochlear nucleus (Zeng et al., 2009). Upregulation of somatosensory inputs to granule cells, which innervate fusiform cells, could explain the increased bimodal enhancement after noise exposure. Interestingly, animal models of neuropathic pain and epilepsy, both characterized by hyperexcitability, show increased VGLUT2 expression (Wallén-Mackenzie et al., 2010), suggesting that upregulation of VGLUT2 expression in auditory centers might lead to hyperexcitability underlying tinnitus. Indeed, increased responsiveness to somatosensory stimulation was demonstrated after broad-band noise exposure and permanent threshold shifts: thresholds and latencies to trigeminal ganglion stimulation were decreased and the response amplitudes 
A
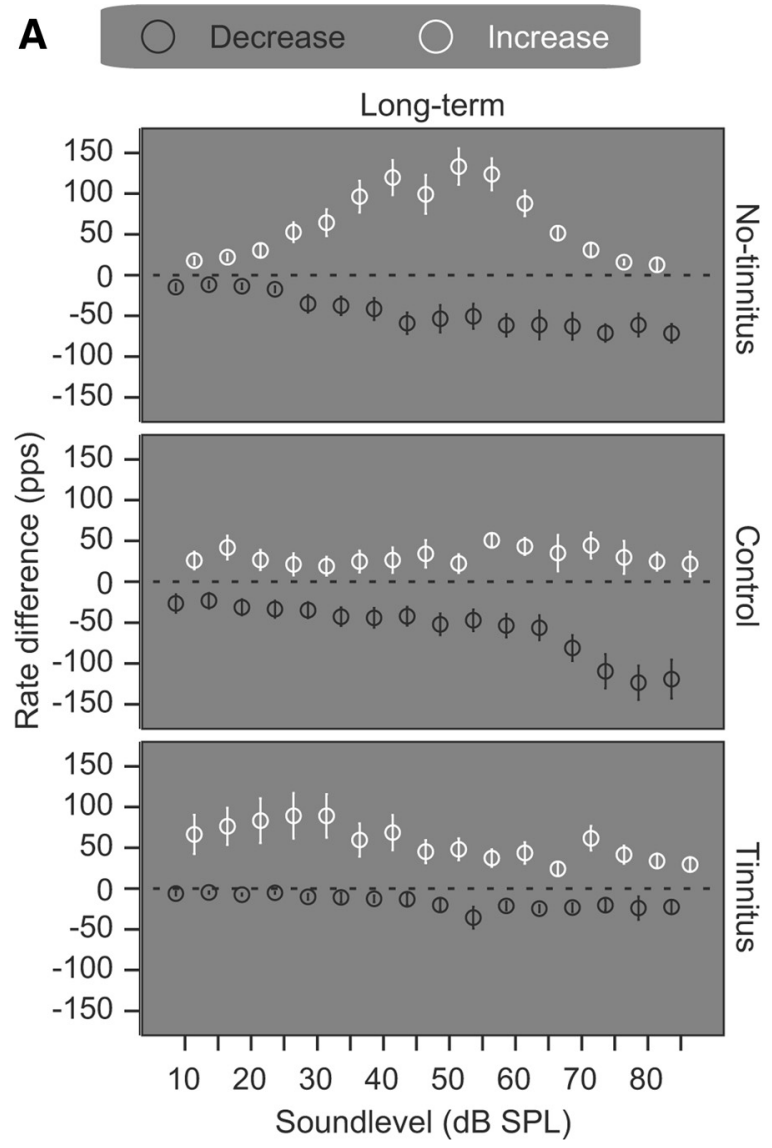

\section{B Number of C Sum of data points changes}
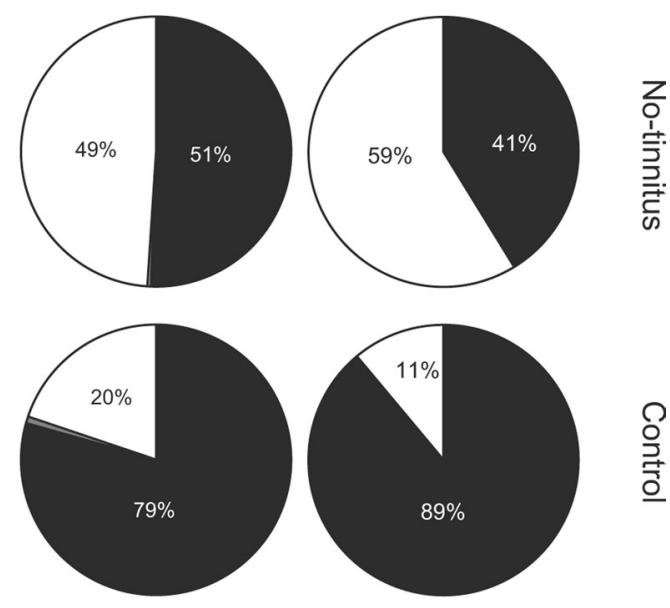

을
음

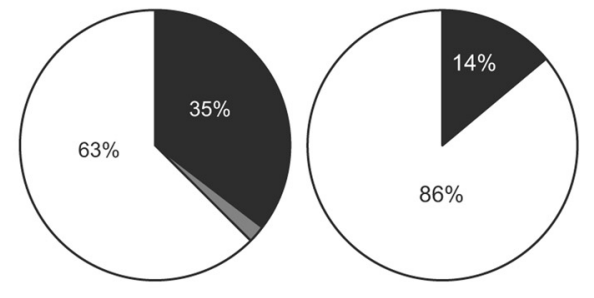

㑒

Figure 14. Chopper units show predominant long-term somatosensory enhancement in tinnitus and no-tinnitus animals. $A$, Long-term rate differences across level: control, $n=5$ animals $/ 23$ RLFs; no-tinnitus, $n=2$ animals/15 RLFs; tinnitus, $n=3$ animals/15 RLFs. All RLFs were recorded at the units' $(F$. $\boldsymbol{B}, \boldsymbol{C}$, Number of data points ( $\boldsymbol{B}$ ) and the summed rate difference ( $\boldsymbol{C}$. Compared are control animals with noise-exposed animals that developed tinnitus or with noise-exposed animals that did not develop tinnitus. Suppression, i.e., a reduction of discharge rate, is shown as black plots or pies and enhancement, i.e., increase of discharge rate, as white plots or pies. For a detailed description see legend of Figure 7.

increased (Shore et al., 2008). These findings are paralleled by heightened responses to somatosensory stimulation in tinnitus patients demonstrated by fMRI (Lanting et al., 2010). Increased excitatory somatosensory drive to DCN neurons could explain why loudness and pitch of tinnitus can be changed by somatosensory stimulation in the face, head and neck regions (Pinchoff et al., 1998; Levine, 1999). If the tinnitus percept is produced by a specific group of neurons with increased sensitivity to somatosensory inputs, somatosensory stimulation would change the response properties of those specific neurons, thereby changing the tinnitus percept.

The shift toward bimodal enhancement occurred in both buildup and chopper units, suggesting an enhancement of somatosensory input across unit types. In contrast, the increases in tone-evoked responses and spontaneous activity were only observed in buildup units. This could be explained by differences in the initial membrane potentials of these two unit types (Kanold and Manis, 1999), leading to differential effects of enhanced somatosensory inputs (Zeng et al., 2009) on sound-driven activity.

An increase in somatosensory-evoked DCN discharges after noise exposure (Shore et al., 2008) should also change the relative timing between presynaptic parallel fiber discharges and postsynaptic discharges of fusiform cells. A higher rate of shorter time delays between presynaptic and postsynaptic events is likely to induce plasticity at the parallel fiber-fusiform cell synapse, which depends on the specific timing between and frequency of pairings of presynaptic and postsynaptic events (Fujino and Oertel, 2003;
Tzounopoulos et al., 2004, 2007). This, in addition to upregulation of VGLUT2-positive synapses, might explain the shift toward bimodal enhancement after noise exposure.

\section{References}

Anari M, Axelsson A, Eliasson A, Magnusson L (1999) Hypersensitivity to sound. Scand Audiol 28:219-230.

Bell CC (1986) Duration of plastic change in a modifiable efference copy. Brain Res 369:29-36.

Bell CC (2001) Memory-based expectations in electrosensory systems. Curr Opin Neurobiol 11:481-487.

Bell CC, Han V, Sawtell NB (2008) Cerebellum-like structures and their implications for cerebellar function. Annu Rev Neurosci 31:1-24.

Bell C, Bodznick D, Montgomery J, Bastian J (1997) The generation and subtraction of sensory expectations within cerebellum-like structures. Brain Behav Evol 50 [Suppl 1]:17-31.

Biesinger E, Reisshauer A, Mazurek B (2008) [The role of the cervical spine and the craniomandibular system in the pathogenesis of tinnitus. Somatosensory tinnitus]. HNO 56:673-677.

Brozoski TJ, Bauer CA, Caspary DM (2002) Elevated fusiform cell activity in the dorsal cochlear nucleus of chinchillas with psychophysical evidence of tinnitus. J Neurosci 22:2383-2390.

Cai S, Ma WL, Young ED (2009) Encoding intensity in ventral cochlear nucleus following acoustic trauma: implications for loudness recruitment. J Assoc Res Otolaryngol 10:5-22.

Dan Y, Poo MM (2006) Spike timing-dependent plasticity: from synapse to perception. Physiol Rev 86:1033-1048.

Dauman R, Bouscau-Faure F (2005) Assessment and amelioration of hyperacusis in tinnitus patients. Acta Otolaryngol 125:503-509.

Doiron B, Zhao Y, Tzounopoulos T (2011) Combined LTP and LTD of 
modulatory inputs controls neuronal processing of primary sensory inputs. J Neurosci 31:10579-10592.

Eggermont JJ (2005) Tinnitus: neurobiological substrates. Drug Discov Today 10:1283-1290.

Eggermont JJ, Roberts LE (2004) The neuroscience of tinnitus. Trends Neurosci 27:676-682.

Fujino K, Oertel D (2003) Bidirectional synaptic plasticity in the cerebellum-like mammalian dorsal cochlear nucleus. Proc Natl Acad Sci U S A 100:265-270.

Haenggeli CA, Pongstaporn T, Doucet JR, Ryugo DK (2005) Projections from the spinal trigeminal nucleus to the cochlear nucleus in the rat. J Comp Neurol 484:191-205.

Itoh K, Kamiya H, Mitani A, Yasui Y, Takada M, Mizuno N (1987) Direct projections from the dorsal column nuclei and the spinal trigeminal nuclei to the cochlear nuclei in the cat. Brain Res 400:145-150.

Kaltenbach JA, Zhang J, Afman CE (2000) Plasticity of spontaneous neural activity in the dorsal cochlear nucleus after intense sound exposure. Hear Res 147:282-292.

Kaltenbach JA, Zacharek MA, Zhang J, Frederick S (2004) Activity in the dorsal cochlear nucleus of hamsters previously tested for tinnitus following intense tone exposure. Neurosci Lett 355:121-125.

Kaltenbach JA, Zhang J, Finlayson P (2005) Tinnitus as a plastic phenomenon and its possible neural underpinnings in the dorsal cochlear nucleus. Hear Res 206:200-226.

Kanold PO, Manis PB (1999) Transient potassium currents regulate the discharge patterns of dorsal cochlear nucleus pyramidal cells. J Neurosci 19:2195-2208.

Kanold PO, Davis KA, Young ED (2011) Somatosensory context alters auditory responses in the cochlear nucleus. J Neurophysiol 105:1063-1070.

Koehler SD, Pradhan S, Manis PB, Shore SE (2011) Somatosensory inputs modify auditory spike timing in dorsal cochlear nucleus principal cells. Eur J Neurosci 33:409-420.

Kujawa SG, Liberman MC (2009) Adding insult to injury: cochlear nerve degeneration after "temporary" noise-induced hearing loss. J Neurosci 29:14077-14085.

Lanting CP, de Kleine E, Eppinga RN, van Dijk P (2010) Neural correlates of human somatosensory integration in tinnitus. Hear Res 267:78-88.

Levine RA (1999) Somatic (craniocervical) tinnitus and the dorsal cochlear nucleus hypothesis. Am J Otolaryngol 20:351-362.

Levine RA, Abel M, Cheng H (2003) CNS somatosensory-auditory interactions elicit or modulate tinnitus. Exp Brain Res 153:643-648.

Levine RA, Nam EC, Oron Y, Melcher JR (2007) Evidence for a tinnitus subgroup responsive to somatosensory based treatment modalities. Prog Brain Res 166:195-207.

Lockwood AH, Salvi RJ, Burkard RF (2002) Tinnitus. N Engl J Med 347:904-910

Middleton JW, Kiritani T, Pedersen C, Turner JG, Shepherd GM, Tzounopoulos T (2011) Mice with behavioral evidence of tinnitus exhibit dorsal cochlear nucleus hyperactivity because of decreased GABAergic inhibition. Proc Natl Acad Sci U S A 108:7601-7606.

Mulders WH, Ding D, Salvi R, Robertson D (2011) Relationship between auditory thresholds, central spontaneous activity, and hair cell loss after acoustic trauma. J Comp Neurol 519:2637-2647.

Nelson JJ, Chen K (2004) The relationship of tinnitus, hyperacusis, and hearing loss. Ear Nose Throat J 83:472-476.

Noreña AJ (2011) An integrative model of tinnitus based on a central gain controlling neural sensitivity. Neurosci Biobehav Rev 35:1089-1109.

Norena A, Micheyl C, Chéry-Croze S, Collet L (2002) Psychoacoustic characterization of the tinnitus spectrum: implications for the underlying mechanisms of tinnitus. Audiol Neurootol 7:358-369.

Oertel D, Young ED (2004) What's a cerebellar circuit doing in the auditory system? Trends Neurosci 27:104-110.

Pfaller K, Arvidsson J (1988) Central distribution of trigeminal and upper cervical primary afferents in the rat studied by anterograde transport of horseradish peroxidase conjugated to wheat germ agglutinin. J Comp Neurol 268:91-108.

Pinchoff RJ, Burkard RF, Salvi RJ, Coad ML, Lockwood AH (1998) Modulation of tinnitus by voluntary jaw movements. Am J Otol 19:785-789.

Roberts LE, Moffat G, Bosnyak DJ (2006) Residual inhibition functions in relation to tinnitus spectra and auditory threshold shift. Acta Otolaryngol Suppl 27-33.
Rubinstein B, Axelsson A, Carlsson GE (1990) Prevalence of signs and symptoms of craniomandibular disorders in tinnitus patients. J Craniomandib Disord 4:186-192.

Sanchez TG, Guerra GC, Lorenzi MC, Brandão AL, Bento RF (2002) The influence of voluntary muscle contractions upon the onset and modulation of tinnitus. Audiol Neurootol 7:370-375.

Schaaf H, Klofat B, Hesse G (2003) [Hyperacusis, phonophobia, and recruitment. Abnormal deviations of hearing associated with hypersensitivity to sound]. HNO 51:1005-1011.

Schaette R, Kempter R (2009) Predicting tinnitus pitch from patients' audiograms with a computational model for the development of neuronal hyperactivity. J Neurophysiol 101:3042-3052.

Shore S, Zhou J, Koehler S (2007) Neural mechanisms underlying somatic tinnitus. Prog Brain Res 166:107-123.

Shore SE (2005) Multisensory integration in the dorsal cochlear nucleus: unit responses to acoustic and trigeminal ganglion stimulation. Eur J Neurosci 21:3334-3348.

Shore SE, Vass Z, Wys NL, Altschuler RA (2000) Trigeminal ganglion innervates the auditory brainstem. J Comp Neurol 419:271-285.

Shore SE, Koehler S, Oldakowski M, Hughes LF, Syed S (2008) Dorsal cochlear nucleus responses to somatosensory stimulation are enhanced after noise-induced hearing loss. Eur J Neurosci 27:155-168.

Stabler SE, Palmer AR, Winter IM (1996) Temporal and mean rate discharge patterns of single units in the dorsal cochlear nucleus of the anesthetized guinea pig. J Neurophysiol 76:1667-1688.

Sun W, Lu J, Stolzberg D, Gray L, Deng A, Lobarinas E, Salvi RJ (2009) Salicylate increases the gain of the central auditory system. Neuroscience 159:325-334.

Turner JG, Parrish J (2008) Gap detection methods for assessing salicylateinduced tinnitus and hyperacusis in rats. Am J Audiol 17:S185-S192.

Turner JG, Brozoski TJ, Bauer CA, Parrish JL, Myers K, Hughes LF, Caspary DM (2006) Gap detection deficits in rats with tinnitus: a potential novel screening tool. Behav Neurosci 120:188-195.

Tzounopoulos T, Kim Y, Oertel D, Trussell LO (2004) Cell-specific, spike timing-dependent plasticities in the dorsal cochlear nucleus. Nat Neurosci 7:719-725.

Tzounopoulos T, Rubio ME, Keen JE, Trussell LO (2007) Coactivation of pre- and postsynaptic signaling mechanisms determines cell-specific spike-timing-dependent plasticity. Neuron 54:291-301.

Vogler DP, Robertson D, Mulders WH (2011) Hyperactivity in the ventral cochlear nucleus after cochlear trauma. J Neurosci 31:6639-6645.

Wallén-Mackenzie A, Wootz H, Englund H (2010) Genetic inactivation of the vesicular glutamate transporter 2 (VGLUT2) in the mouse: what have we learnt about functional glutamatergic neurotransmission? Ups J Med Sci 115:11-20.

Wang H, Brozoski TJ, Turner JG, Ling L, Parrish JL, Hughes LF, Caspary DM (2009) Plasticity at glycinergic synapses in dorsal cochlear nucleus of rats with behavioral evidence of tinnitus. Neuroscience 164:747-759.

Yang G, Lobarinas E, Zhang L, Turner J, Stolzberg D, Salvi R, Sun W (2007) Salicylate induced tinnitus: behavioral measures and neural activity in auditory cortex of awake rats. Hear Res 226:244-253.

Zeng C, Nannapaneni N, Zhou J, Hughes LF, Shore S (2009) Cochlear damage changes the distribution of vesicular glutamate transporters associated with auditory and nonauditory inputs to the cochlear nucleus. J Neurosci 29:4210-4217.

Zeng C, Shroff H, Shore SE (2011) Cuneate and spinal trigeminal nucleus projections to the cochlear nucleus are differentially associated with vesicular glutamate transporter-2. Neuroscience 176:142-151.

Zhan X, Pongstaporn T, Ryugo DK (2006) Projections of the second cervical dorsal root ganglion to the cochlear nucleus in rats. J Comp Neurol 496:335-348.

Zhao Y, Rubio M, Tzounopoulos T (2011) Mechanisms underlying inputspecific expression of endocannabinoid-mediated synaptic plasticity in the dorsal cochlear nucleus. Hear Res 279:67-73.

Zhou J, Shore S (2004) Projections from the trigeminal nuclear complex to the cochlear nuclei: a retrograde and anterograde tracing study in the guinea pig. J Neurosci Res 78:901-907.

Zhou J, Nannapaneni N, Shore S (2007) Vesicular glutamate transporters 1 and 2 are differentially associated with auditory nerve and spinal trigeminal inputs to the cochlear nucleus. J Comp Neurol 500:777-787. 